\title{
El resveratrol y la 6-bencil amino purina reducen la pérdida de firmeza y color en poscosecha de guanábana (Annona muricata L., Annonaceae)
}

Resveratrol and 6-benzyl amino purine reduce the rate of firmness and weight loss in soursop (Annona muricata L., Annonaceae) postharvest

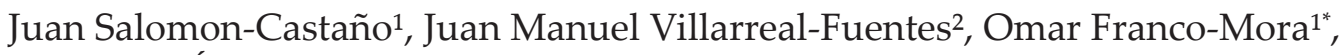 \\ Álvaro Castañeda-Vildózola ${ }^{3}$, Jesús Ricardo Sánchez-Pale ${ }^{3}$
}
${ }^{1}$ Laboratorio de Horticultura, Facultad de Ciencias Agrícolas, Campus Universitario El Cerrillo, Universidad Autónoma del Estado de México (UAEM), Carretera Toluca-Ixtlahuaca km 15.5, El Cerrillo, Piedras Blancas, 50200, Toluca, Estado de México, México.
${ }^{2}$ Facultad de Ciencias Agrícolas, Universidad Autónoma de Chiapas, Entronque carretera Costera y Pueblo de Huehuetán, 30660 , Huehuetán, Chiapas, México.
${ }^{3}$ Facultad de Ciencias Agrícolas (UAEM).

*Autor para correspondencia: ofrancom@uaemex.mx

Fecha de recepción

7 de enero de 2020

Fecha de aceptación

14 de marzo de 2020

Disponible en línea

16 de abril de 2020

Este es un artículo en acceso abierto que se distribuye de acuerdo a los términos de la licencia Creative Commons

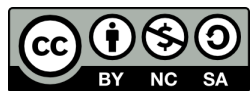

ReconocimientoNoComercial-CompartirIgual 4.0 Internacional

\section{RESUMEN}

Principalmente por su naturaleza climatérica, en guanábana (Annona muricata L., Annonaceae) existen pérdidas de $60 \%$ de la producción durante la comercialización. El objetivo de este trabajo fue evaluar el efecto poscosecha de la aplicación precosecha de una solución acuosa de $1.6 \mathrm{mM}$ de resveratrol (RVS) y $1.0 \mathrm{mM}$ de 6-bencil amino purina (BAP), en frutos de guanábana del Soconusco, Chiapas, México. Se efectuó la aplicación de RVS y BAP o de agua corriente, como control, a frutos de 12 árboles, 10 días antes de cosecha. Una vez cosechados, dichos frutos se almacenaron a $18^{\circ} \mathrm{C} \pm 2$, y se evaluó color, firmeza, contenido de compuestos fenólicos y porcentaje de sólidos solubles totales. Los frutos tratados con RVS y BAP duraron 3 días más en almacenamiento que los frutos control. Además, a 5 días de almacenamiento, los frutos tratados con RVS y BAP conservaron $70 \%$ de firmeza inicial de cosecha, mientras que los frutos control sólo mantuvieron 30\%. La mayoría de los parámetros de color mostraron diferencias significativas entre tratamientos a los 5 y 8 días de almacenamiento: menor cambio de color verde y menor aparición de color marrón en la cáscara de los frutos a los que se aplicó RVS y BAP.

PALABRAS CLAVE

cáscara, climatérico, bioregulador vegetal, pigmento

\section{ABSTRACT}

Mainly due to its climacteric behavior, soursop fruit (Annona muricata L., Annonaceae) loss in fruit production is nearly $60 \%$ during marketing. The objective of the present work was to evaluate the postharvest effect of preharvest application of an aqueous solution of $1.6 \mathrm{mM}$ resveratrol (RVS) and $1.0 \mathrm{mM}$ 6-benzyl amino purine (BAP), in soursop fruits from Soconusco, Chiapas, Mexico. RVS and BAP or tap water as control were applied to fruits of 12 soursop trees, 10 days before harvest. Then, those fruits were harvested and storage at $18{ }^{\circ} \mathrm{C} \pm 2$ and kinetic of color, peel firmness, the content of phenolic compounds, and the percentage of total soluble solids were evaluated. Fruits treated with RVS and BAP presented 3 days more of storage period in relation to control. Moreover, at day 5 of storage, the soursop fruit treated with RVS and BAP conserved $70 \%$ of initial firmness at harvest, whereas control fruit preserved only 30\%. Generally, color parameters showed differences between treatments at 5 and 8 days in storage; it suggests less change in initial peel green color and reduced peel browning by the effect of RVS and BAP application.

KEYWORDS

peel, climateric, plant bioregulator, plant pigment 


\section{INTRODUCCIÓN}

El guanábano (Annona muricata L., Annonaceae) es un frutal originario de las regiones tropicales de América del Sur. Se desarrolla en varios países del Caribe, Centroamérica, Sudamérica, sureste de China, Australia, África occidental e Islas del Pacífico (Cuadros 2008). México es el mayor productor de esta fruta, dicha producción ocurre en Nayarit, Colima, Veracruz y Guerrero (Evangelista-Lozano et al. 2003; São José et al. 2014; SADER 2020).

El fruto es de forma irregular, presenta residuos estilares espiniformes, lo cual hace difícil su manipulación y predispone daños mecánicos en la poscosecha (Ploetz 2003). Los factores previos, más la presencia de enfermedades poscosecha, generan pérdidas de hasta $60 \%$ de la producción (Espinosa et al. 2013), lo cual limita su distribución y comercialización en fresco. Por su naturaleza climatérica, durante la etapa de maduración de la guanábana se presentan ciclos de respiración y producción de etileno, con máximos entre 100 y $350 \mathrm{ml} \mathrm{kg}^{-1} \mathrm{~h}^{-1} \mathrm{de}$ $\mathrm{CO}_{2}$, así como 250 y $350 \mathrm{ml} \mathrm{kg}^{-1} \mathrm{~h}^{-1}$ de etileno (Worrell et al. 1994), lo que reduce la vida de anaquel, la cual es de máximo 9 días, aproximadamente (Pareek et al. 2011). En el almacenamiento ocurren cambios físicos, químicos y bioquímicos que provocan el deterioro de la calidad; principalmente, oscurecimiento, ablandamiento y ruptura de la cáscara, aspectos que favorecen el ataque de hongos (Ploetz 2003).

Actualmente, las tecnologías poscosecha disponibles para guanábana son capaces de mantener la vida de anaquel por aproximadamente 9 días. La cadena de frío es una alternativa para la conservación de productos frescos; sin embargo, la guanábana es susceptible a daños por frío cuando se somete a temperaturas inferiores a $15{ }^{\circ} \mathrm{C}$ (Reginato y Lizana 1980). Castillo-Ánimas et al. (2005) reportan que con la aplicación de ceras no se evitó el daño por frío en guanábanas cosechadas en dos estados de madurez y almacenadas a temperaturas menores de $18{ }^{\circ} \mathrm{C}$. Por otro lado, Lima et al. (2010) mencionan que la aplicación de $200 \mathrm{~nL} \mathrm{~L}^{-1}$ de 1-MCP a frutos de guanábana y almacenados a $15{ }^{\circ} \mathrm{C}$ no prolongó la vida de anaquel por más de 8 días. Sin embargo, el uso de ceras en combinación con 1-MCP ha reducido la pérdida de firmeza y aumentado el porcentaje de sólidos solubles totales (SST) (Lima y Alves 2011). Tovar-Gómez et al. (2011) indicaron que la aplicación de emulsiones de cera de carnauba tipo III, carnauba con aceites siliconados y candelilla en combinación con 1-MCP aplicado en guanábanas, disminuyeron la pérdida de peso, alcanzando la madurez organoléptica a los 15 días de almacenamiento. Por su parte, Moreno-Hernández et al. (2014) mencionan que la combinación de 1-MCP con emulsiones de candelilla mantuvo la calidad nutricional y hortícola de la guanábana de 14 a 15 días en almacenamiento a $16{ }^{\circ} \mathrm{C}$.

Por otra parte, como alternativa para mejorar la vida poscosecha de frutos de la familia anonácea, se ha empleado la aplicación de hormonas y bioreguladores vegetales. La 6-bencil amino purina (BAP) está involucrada en el control y regulación de la maduración de frutos (Giovannoni 2001), al reducir la tasa de degradación de pigmentos y proteínas fotosintéticas y mantener el equilibrio hídrico de tejidos vegetales (Costa et al. 2005; Zavaleta-Mancera et al. 2007). Por ejemplo, en poscosecha de brócoli (Brassica oleracea L.), la BAP disminuyó la tasa de respiración y retrasó el amarillamiento de la inflorescencia (Zhu et al. 2004; Zaicovski et al. 2008). La aplicación de esta hormona también mantuvo el contenido de clorofila y evitó la deshidratación en la cáscara durante la maduración de plátano (Musa x paradisiaca L.) (Aghofack-Nguezemi y Manka'abiengwa 2012).

El resveratrol (RVS) es una fitoalexina y constituye uno de los 300 salvestroles de las plantas. Su aplicación precosecha o poscosecha incrementa la calidad sensorial, la vida de almacenamiento y la calidad nutricional en frutos de manzana (Malus comunis L.), aguacate (Persea americana Mill.), tomate (Solanum lycopersicum L.), pimiento (Capsicum annuum L.), fresa (Fragaria vesca L.) y uva (Vitis vinifera L.) (Jiménez et al. 2005). En mandarina (Citrus reticulata Blanco), el RVS conservó el color de la cáscara durante 12 semanas a $10{ }^{\circ} \mathrm{C}$ (Cherukuri, 2007). Morales et al. (2014) indicaron que la aplicación de RVS entre 8 y 15 días previos a la cosecha de frutos de chirimoya (Annona cherimola Mill.) 'Fino de Jete' y 'Bronceada' redujo la tasa de ablandamiento durante 15 días de almacenamiento. Franco-Mora et al. (2015) observaron que frutos de chirimoya tratados en precosecha de manera simultánea con RVS y BAP disminuyeron su tasa de cambio de color y pérdida de firmeza de pulpa y peso de fruto en comparación con frutos control durante 15 días. Por los antecedentes indicados, el objetivo de este trabajo fue evaluar el efecto de la aplicación en precosecha de una solución acuosa de $1.6 \mathrm{mM}$ de resveratrol y $1.0 \mathrm{mM}$ de 6-bencil amino purina sobre la tasa de pérdida de firmeza y cambio de color durante el almacenamiento poscosecha de guanábana. 


\section{MATERIALES Y MÉTODOS}

El sitio experimental, huerta comercial, se ubicó en la finca "La Libertad" (15॰ 5' 12" N, 92 15' 7" O, 800 msnm), ejido 26 de Octubre, municipio de Tapachula, Chiapas, México. Se emplearon los frutos de 12 árboles de guanábano, y los tratamientos se aplicaron sólo a los frutos que, por su apariencia visual - determinada por el productor-, serían cosechados 10 días después de la aplicación. Previo a la aplicación, los frutos que se tratarían se marcaron consecutivamente, para aplicar bioreguladores o agua. La solución de bioreguladores se llevó a cabo como lo indican Salomon-Castaño et al. (2020): en agua corriente se disolvió $1.6 \mathrm{mM}$ de RVS (Laboratorios Lemi) y $1.0 \mathrm{mM}$ de BAP (SIGMA). Dicha solución, o el agua corriente, se aplicó con una brocha a los frutos, cubriendo toda su superficie de acuerdo con la marcación descrita previamente. Durante la aplicación se colocó una franela para evitar escurrimiento de la solución en frutos adyacentes.

Después de 10 días de haberse efectuado la aplicación, sólo se cosecharon frutos cuyo índice de corte - basados en la experiencia del productor-, implicando tonalidad de la cáscara y la pérdida de rigidez de los rudimentos estilares, fueran homogéneos en la maduración de cosecha. Para cada tratamiento se manejaron 30 frutos, los cuales se trasladaron al Laboratorio de Poscosecha de la Facultad de Ciencias Agrícolas de la Universidad Autónoma de Chiapas. Los frutos fueron almacenados a $18{ }^{\circ} \mathrm{C}$, por $1,5,8$ y 11 días después de la cosecha (DDC), y se registraron color, peso, firmeza y porcentaje de sólidos solubles totales (SST). Una porción de pulpa de los frutos empleados se conservó a $-20{ }^{\circ} \mathrm{C} y$, posteriormente, dichas muestras se trasladaron al Laboratorio de Horticultura de la Facultad de Ciencias Agrícolas de la Universidad Autónoma del Estado de México, donde se determinó el contenido de compuestos fenólicos. Para cada uno de los muestreos se emplearon siete repeticiones por tratamiento, un fruto por repetición.

\section{FirMeza de LA CÁSCARA}

La firmeza de cáscara se evaluó con un texturómetro Chatillon DFE-050 (Ametek, EUA), equipado con un puntal cónico de $0.7 \mathrm{~cm}$ de diámetro, con una penetración de $5 \mathrm{~mm}$ de profundidad a $5 \mathrm{~mm} / \mathrm{s}$ en 3 diferentes puntos de la superficie de los frutos; los resultados se reportaron en Newtons (N). El fin del almacenamiento respectivo se determinó cuando el resultado era igual o menor a $5 \mathrm{~N}$.

\section{Color}

Se determinó sobre la cáscara del fruto con un espectrofotómetro Ci60 (X-rite, EUA), programado con tres disparos por fruto en diferentes lugares de la cáscara. Los valores reportados son en escala de CIELab (L, $a^{*}, b^{*}, c y$ h). El índice de color (IC) fue calculado con la siguiente fórmula: $\left[\left(a^{*} \times 1000\right) /(L x\right.$ b*)] (García et al. 2011).

\section{Sólidos SOlubles TOTALES}

Cada fruto se dividió en tres secciones, de manera longitudinal, y de cada una de ellas se tomó pulpa sin semilla, y se incorporaron 3 gotas de jugo de manera directa al lector del refractómetro 300034 (Sper SCi., EUA). La media de las tres mediciones por fruto se tomó como el resultado de cada fruto, y se expresó en grados brix $\left({ }^{\circ} \mathrm{Bx}\right)$.

\section{Compuestos fenólicos}

Se pesaron $2 \mathrm{~g}$ de pulpa de guanábana y se maceraron completamente con $40 \mathrm{ml}$ de etanol a $80 \%$. Dicha solución fue sometida a baño maría por 5 minutos; luego, se dejó enfriar a temperatura ambiente, se filtró y se guardó en botellas de plástico de $15 \mathrm{ml}$, para almacenarse a $-20^{\circ} \mathrm{C}$, por no más de $48 \mathrm{~h}$. Para la determinación de los compuestos fenólicos, se utilizó el método de Folín-Ciocalteu descrito por Waterman y Mole (1994). Los resultados fueron expresados en miligramos de equivalentes de ácido tánico por gramo de peso fresco $\left(\mathrm{mg} \mathrm{EAT} \mathrm{g}^{-1} \mathrm{PF}\right)$.

\section{Análisis estadístico}

Los resultados se analizaron con un diseño completamente al azar y se compararon los tratamientos con la prueba t de student a un nivel de significancia de $\mathrm{p} \leq 0.05$ en el software SPSS (Statistical Package Social Science), versión 2012, y las gráficas se elaboraron con el paquete Sigma Plot 2010 (Systat, 2012).

\section{RESUltados Y Discusión Periodo de almacenamiento}

Los frutos tratados con RVS y BAP tuvieron tres días más de almacenamiento (11 días), en relación con los frutos control (8 días) (Figura 1), resultados similares a los observados por Salomon-Castaño et al. (2020) al aplicar, 15 días antes de la cosecha de chirimoya 
"Bays", los bioreguladores RVS y BAP. El aumento de la vida poscosecha y el mantenimiento de la calidad del productoesunamaneradecontribuiraladisponibilidad de los alimentos sin necesidad de incrementar la producción en campo (Kusumaningrum et al. 2015), lo cual disminuye el desperdicio de productos frutícolas. Entre los métodos inocuos para incrementar la vida poscosecha de guanábana se encuentra la aplicación de 1-MCP (Montalvo et al. 2014); en este sentido, la aplicación de RVS y BAP también es inocua, y agrega la facilidad de aplicación, ya que puede efectuarse con mochila aspersora en el huerto previo a la cosecha (Salomon-Castaño et al. 2020). A diferencia de los trabajos de Morales et al. (2014 y 2016) y Franco-Mora et al. (2015), en este experimento, la fuente de RVS no fue reactivo puro, sino una fuente disponible en farmacias comerciales.

\section{FirmezA}

Con la aplicación simultánea de RVS y BAP, a los días 5 y 8 de almacenamiento, la firmeza de cáscara fue mayor ( $\mathrm{p} \leq 0.05)$ que en los frutos control. Por otro lado, al día 5 después de cosecha, los frutos control disminuyeron $70 \%$ de la firmeza inicial, mientras que los tratados con RVS y BAP mostraron pérdida de $30 \%$. Sin embargo, a los 8 días de almacenamiento y con la continua tendencia decreciente de la firmeza en los frutos de ambos tratamientos, la pérdida de firmeza fue de $90 \%$ para el control y $87 \%$ para los tratados con los biorreguladores (Figura 1). Resultados similares son reportados por Espinosa et al. (2013), en frutos de guanábana tratada con 1-MCP, quienes presentaron alrededor de $100 \mathrm{~N}$ de diferencia con el control al día 5 y 6 de almacenamiento, pero a partir del séptimo día y hasta terminar su vida poscosecha ya no hubo diferencia estadística. Además, de acuerdo con Márquez et al. (2012) y Espinosa et al. (2013), al final del almacenamiento de guanábana se observan valores de firmeza de pulpa cercanos a $5 \mathrm{~N}$.

En guanábana se ha determinado que el rápido ablandamiento de los frutos durante la maduración coincide con la mayor actividad de la amilasa, pectinmetilesterasa, poligalacturonasa y la ßs-galactosidasa de la pared celular (Lima et al. 2006). En este sentido, la reducción en la tasa de ablandamiento de pulpa en 2 cultivares de chirimoya, por la aplicación precosecha de RVS y BAP, se atribuyó a la disminución de la actividad de la ß-galactosidasa y al retraso de la actividad de la poligalacturonasa en relación con los frutos control, de tal manera que la aplicación conserva la integridad de las células (Franco-Mora et al. 2015). Por otro lado, de manera particular, el RVS está relacionado con la lignificación de la pared celular (Van Buren 1986; Morales et al. 2016). Los conocimientos previos generados en poscosecha de anonáceas con la aplicación de RVSy BAP, contribuyen a explicar el menor ablandamiento observado en cáscara de guanábana del Soconusco, Chiapas. Limitar o retrasar el ablandamiento de la guanábana en poscosecha puede aumentar la posibilidad de su manejo, transporte y comercialización, reduciendo los altos porcentajes de pérdida económica durante la etapa poscosecha de este fruto (Espinosa et al. 2013).

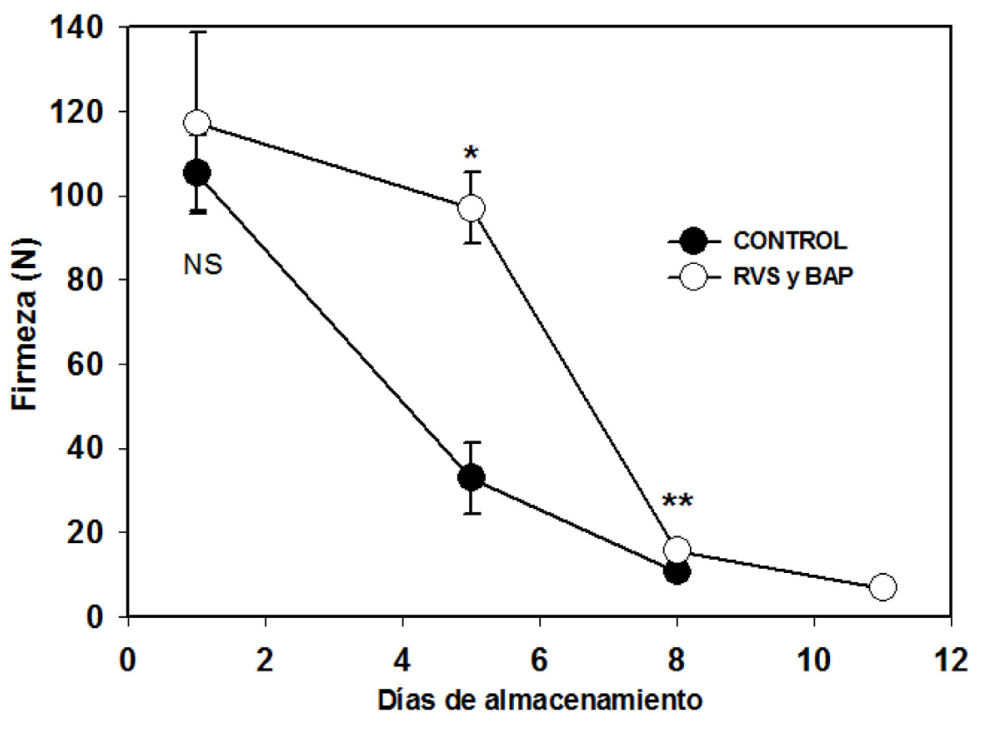

Figura 1. Firmeza de la cáscara de guanábana tratada con resveratrol y 6-bencil amino purina. Los datos son la media de 7 observaciones \pm error estándar. NS, no significativo; ${ }^{*}$ significativo con la prueba $t$ de student a $0.05 ;{ }^{* *}$ significativo con la prueba $t$ de student a 0.01 . 


\section{LUMINOSIDAD (L)}

A los 8 días de almacenamiento, la luminosidad de la cáscara de guanábana fue diferente $(\mathrm{p} \leq 0.05)$ entre tratamientos; los frutos tratados con RVS y BAP redujeron $5 \%$ su valor inicial, mientras que los frutos control descendieron $8 \%$ del valor presentado al inicio del almacenamiento (Cuadro 1). Márquez (2009) menciona que dicha pérdida de luminosidad se relaciona con la sobremadurez e inicio de la senescencia, así como el oscurecimiento de la cáscara.

\section{$\mathbf{A}^{*}$}

Para el parámetro de color $\mathrm{a}^{*}$, a los 5 y 8 días después de cosecha, se presentó diferencia significativa $(\mathrm{p} \leq 0.05)$, pero, cada día, uno de los dos tratamientos tuvo el mayor valor (Cuadro 1). Al día 8 de almacenamiento, los frutos control se acercaron más, en relación con los frutos tratados con RVS y BAP, al cero, lo cual significa mayor pérdida del color verde de la cáscara. En tal sentido, se aprecia que los frutos tratados con RVS y BAP tuvieron estabilidad en este parámetro del color después del día 5 de cosecha. Esta estabilidad es importante, ya que, como indicó Márquez (2009), generalmente, los frutos de guanábana al final de su periodo poscosecha alcanzan valores positivos del factor $\mathrm{a}^{*}$, lo que indica degradación del pigmento verde. De la misma manera, la aplicación de BAP y RVS redujo la degradación de color verde en la cáscara de chirimoya 'Fino de Jete' y 'Ruth' (Franco-Mora et al. 2015). En este sentido, se ha sugerido que BAP es el responsable de esta acción, ya que, previamente, RVS aplicado de manera única no había mantenido la tonalidad verde de la cáscara de chirimoya (Morales et al. 2014). Complementando esta idea, en la cáscara de banano, la aplicación de BAP redujo la pérdida de clorofila al disminuir la actividad de la clorofilasa (Yang et al. 2009; Aghofack-Nguezemi y Manka'abiengwa 2012).

Cuadro 1. Parámetros e índice de color en cáscara de guanábana tratada con resveratrol y 6-bencil amino purina.

\begin{tabular}{|c|c|c|c|c|c|c|c|c|c|c|c|}
\hline & \multicolumn{3}{|c|}{ Día 1} & \multicolumn{3}{|c|}{ Día 5} & \multicolumn{2}{|c|}{ Día 8} & & \multicolumn{2}{|c|}{ Día 11} \\
\hline & Control & RVS & & Control & RVS & & Control & RVS & & Control & RVS \\
\hline & & BAP & & & BAP & & & BAP & & & BAP \\
\hline L & 40.7 & 40.6 & NS & 40.4 & 40.3 & NS & 37.6 & 38.4 & * & NA & 38.3 \\
\hline$a^{*}$ & -11.4 & -12.4 & NS & -10.4 & -9.8 & * & -7.0 & -9.8 & $* *$ & NA & -9.1 \\
\hline C & 20.1 & 21.6 & NS & 19.5 & 18.9 & * & 15.4 & 18.9 & $* *$ & NA & 18.0 \\
\hline $\mathbf{h}$ & 110.1 & 110.0 & NS & 106.0 & 109.5 & $* *$ & 105.0 & 109.2 & * & NA & 106.6 \\
\hline IC & -19.3 & -20.4 & NS & -17.3 & -19.4 & $* *$ & -15.5 & -18.9 & $*$ & NA & -17.4 \\
\hline
\end{tabular}

Los datos son la media de 7 frutos, tres lecturas por fruto. NS, no significativo, ${ }^{*}$ significativo al $0.05 ;{ }^{* *}$ significativo al 0.01 con la prueba t de student. NA: sin datos.

\section{C (CROMA)}

Existiópérdidadecromaticidadenambostratamientos, pero sólo ocurrió hasta el día 5 de almacenamiento en los frutos tratados con RVS y BAP, aunque fue mayor $(13 \%)$ que el observado en los frutos control (3\%). Al día 8 de almacenamiento, en el control disminuyó $23 \%$ con respecto al valor de cosecha, mientras que en los frutos tratados con RVS-BAP fue estable desde el día 5. Al día 11 de almacenamiento, en los frutos tratados con RVS-BAP se registró disminución total de $16.8 \%$ con respecto al valor inicial. Lima et al. (2003) mencionan que, en guanábana, la disminución en los valores de los parámetros de color, entre ellos cromaticidad, puede ser atribuida a la degradación de la clorofila por acción enzimática; en este sentido, para este trabajo se sugiere una acción antagonista a la clorofilasa por parte de los biorreguladores aplicados (Aghofack-Nguezemi y Manka'abiengwa 2012).

\section{Hue (H)}

Los valores para hue (110-116) de la guanábana del Soconusco, Chiapas, están por debajo de los reportados para Nayarit, 151.7-164.9 (Jiménez-Zurita et al. 2016) y Malasia, 123-110 (Siang et al. 2019), pero son similares a los reportados en Morelos, 110 (Evangelista-Lozano et al. 2003). Diferencias en los parámetros de color pueden deberse a los diferentes materiales evaluados y a las condiciones edafoclimáticas bajo las cuales se desarrolla el fruto. Para este trabajo, el efecto de RVS y BAP en la cáscara también fue observado sobre la variable $h$; a los días 5 y 8 de almacenamiento poscosecha, los frutos control disminuyeron sus valores, mientras que los frutos con RVS-BAP prácticamente los mantuvieron. Aunque se reportó menor pérdida de valor para $\mathrm{h}$ en la cáscara de guanábana por efecto del 1-MCP (Siang et al. 2019), en este trabajo, la estabilidad de este valor por efecto de RVS-BAP es de importancia, ya que la 
pérdida de color verde de la cáscara es uno de los cambios visibles y notorios de la maduración de guanábana como consecuencia de la degradación de la clorofila y la descomposición del cloroplasto que libera polifenol oxidasas, lo que provoca la oxidación y polimerización de los fenoles (Paull et al. 1983).

\section{ÍNDICE DE COLOR (IC)}

En los frutos control, a medida que avanza el proceso de maduración, se incrementó el índice de color. Sin embargo, en los frutos tratados con RVS y BAP, dicho índice se mantuvo los primeros 8 días de almacenamiento, $\mathrm{y}$, aunque a 11 días de almacenamiento aumentó, no alcanzó los niveles de los frutos control. De acuerdo con la escala de índice de color de Vignoni et al. (2006), los resultados observados en el almacenamiento poscosecha clasifican a los frutos control control aumentaron su nivel de fenoles, regresando prácticamente a su nivel del corte, los frutos tratados con RVS-BAP mantuvieron los niveles del día 5, durante los días 8 y 11 de almacenamiento (Figura 2). La concentración de los compuestos fenólicos depende de factores como cultivar, estacionalidad, condiciones vegetativas (especialmente el contenido de nutrimentos y la intensidad de la energía solar), estado de salud y madurez de la fruta, procesos de lignificación o deslignificación de las paredes celulares (Julián 2009); además, se ha sugerido que las diferencias en los procedimientos de extracción influyen en los resultados (Siang et al. 2019).

Para este trabajo, la mayor concentración fenólica en frutos control, a 8 días de almacenamiento, coincide con su último día de anaquel y corresponde a los menores valores de IC y L, sugiriendo su relación con cambios de color en la cáscara, posiblemente por oxidación (Paull

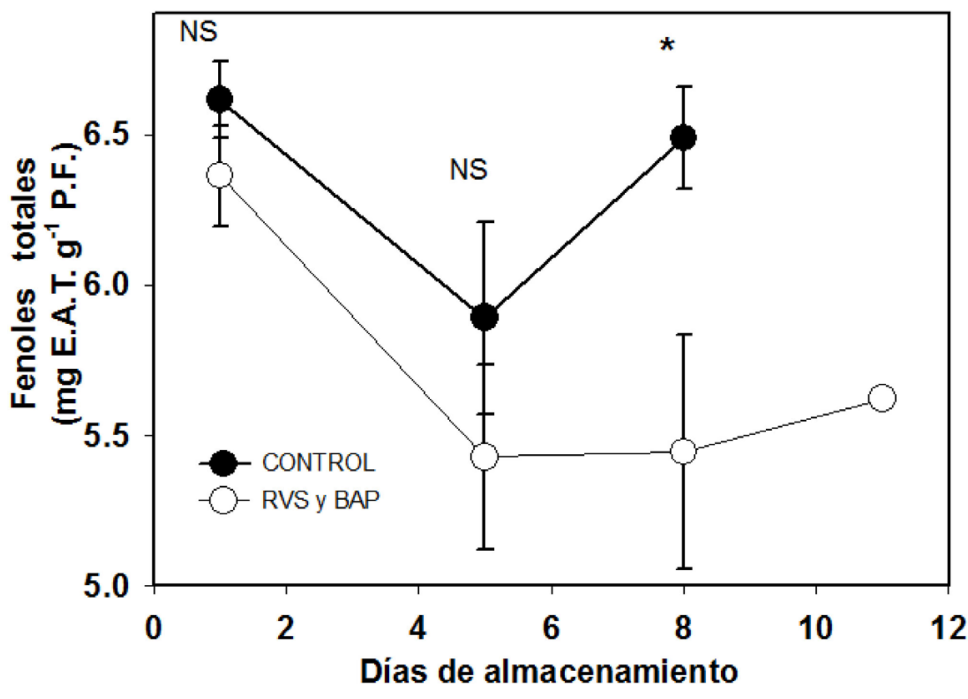

Figura 2. Contenido de compuestos fenólicos en poscosecha de frutos de guanábana tratados con resveratrol y 6-bencil amino purina. NS, no significativo; * significativo con la prueba $t$ de student a 0.05 . Los datos son la media de 7 observaciones \pm error estándar.

en "verde profundo" al inicio, para finalizar en "verde amarillento" (valores de IC de -20 a -2), mientras que el uso de RVS y BAP conservó el color verde inicial de las guanábanas, manteniendo este criterio de calidad.

\section{Compuestos fenólicos}

Al día 5 de almacenamiento hubo disminución del contenido de compuestos fenólicos, en ambos tratamientos, $y$, posteriormente, aumentó en diferente proporción, lo cual generó diferencia estadística a los 8 días de almacenamiento. Mientras que los frutos et al. 1983). Siang et al. (2019) observaron diferentes comportamientos en la cinética del contenido de compuestos fenólicos en guanábana tratada con 4 dosis de 1-MCP. Dichos autores indicaron que, en el control, la reducción del contenido de estos compuestos se relaciona con el término de la madurez completa.

\section{Porcentaje de sólidos solubles totales (SST)}

Para el porcentaje de SST, la aplicación precosecha de RVS y BAP no generó diferencia estadística $(p<0.05)$ con respecto al control al momento del corte (Figura 3). 


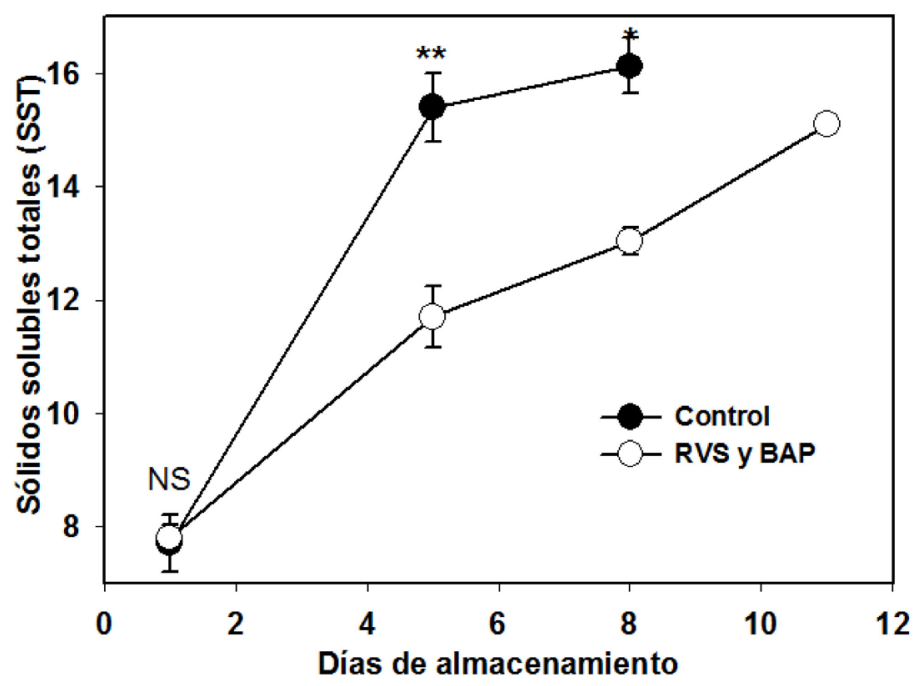

Figura 3. Concentración de sólidos solubles totales en poscosecha de frutos de guanábana con aplicación de resveratrol y 6-bencil amino purina. NS, no significativo; * significativo con la prueba $t$ de student a 0.05 ; ${ }^{* *}$ significativo con la prueba $t$ de student a 0.01 . Los datos son la media de 7 observaciones \pm error estándar.

Sin embargo, para los días 5 y 8 de almacenamiento, los frutos control presentaron mayor $(p<0.05)$ porcentaje de SST que los frutos tratados con RVS y BAP. Al final de 11 días de almacenamiento, los frutos tratados con RVS y BAP no alcanzaron $16^{\circ}$ Brix, contenido que se presentó a los 8 días de almacenamiento en los frutos control. Morales et al. (2016) indicaron que la aplicación única de RVS limitó la pérdida de firmeza de cáscara y pulpa en chirimoya 'Fino de Jete', pero no influyó en el porcentaje de SST. En el caso de las guanábanas de este trabajo, la menor pérdida de color sí estuvo acompañada con menor acumulación de SST, lo cual puede indicar menor metabolismo de maduración y senescencia en los frutos tratados con RVS y BAP en relación con los frutos control (Martínez-González et al. 2017). Por su parte, Siang et al. (2019) indicaron que menor tasa de respiración y producción de etileno no se relacionó con menor porcentaje de SST en guanábanas tratadas con 1-MCP.

\section{Conclusiones}

La aplicación, 10 días antes de la cosecha, de una solución de $1.6 \mathrm{mM}$ de resveratrol y $1.0 \mathrm{mM}$ de 6-bencil amino purina a frutos de guanábana aumentó la vida de anaquel, a $18^{\circ} \mathrm{C}$, por 3 días, en relación con frutos control. Esta combinación de biorreguladores vegetales redujo la pérdida de firmeza de la cáscara, los cambios en parámetros e índice de color y la acumulación de sólidos solubles totales.

\section{Agradecimientos}

Se agradece la beca doctoral del CONACYT al primer autor y el apoyo de los productores de guanábana de la finca "La Libertad". 


\section{LITERATURA CITADA}

Aghofack-Nguezemi J, Manka'abiengwa J. 2012. Effects of exogenously applied benzylaminopurine and kinetin on the ripening of banana (Musa acuminate Colla var. William) fruits. American Journal of Plant Physiology 7: 154-163.

Castillo-Ánimas D, Varela-Hernández G, Pérez-Salvador BR, Pelayo-Zaldívar C. 2005. Daños por frío en guanábana. Índice de corte y tratamientos postcosecha. Revista Chapingo Serie Horticultura 11: 51-57.

Cherukuri K. 2007. Effect of trans-resveratrol treatment on shelf-life and bioactive compounds in Satsuma mandarin. Ph. D. Thesis. Auburn University. Alabama, USA. [Consultado 12 de octubre de 2019]. Disponible en: http://etd.auburn.edu/handle/10415/1344.

Costa ML, Civello PM, Chávez AR, Martínez GA. 2005. Effect of ethephon and 6-benzylaminopurine on chlorophyll degrading enzymes and a peroxidase-linked chlorophyll bleaching during postharvest senescence of broccoli (Brassica oleracea L.) at $20^{\circ} \mathrm{C}$. Postharvest Biology and Technology 35: 191-199. https://doi.org/10.1116/j.postharvbio.2004.07.007

Cuadros V. 2008. Guanábana: Manejo del cultivo y poscosecha. Proexant. Quito, Ecuador.

Espinosa I, Ortiz RI, Tovar B, Mata M, Montalvo E. 2013. Physiological and physicochemical behavior of soursop fruits refrigerated with 1-methylcyclopropene. Journal of Food Quality 36: 10-20. https://doi. org/10.1111/jfq.12011

Evangelista-Lozano S, Cruz-Castillo JG, Pérez-González S, Mercado-Silva E, Dávila-Ortíz G. 2003. Producción y calidad frutícola de guanábanos (Annona muricata L.) provenientes de semilla de Jiutepec, Morelos, México. Revista Chapingo Serie Horticultura 9: 69-79.

Franco-Mora O, Morales-Pérez AA, Castañeda-Vildózola A, Morales-Rosales EJ, Sánchez-Pale JR. 2015. Sprays mixing resveratrol and benzylaminopurine previous harvest helps to preserve postharvest quality in cherimoya. Journal of Agriculture and Life Sciences 2: 1624.

García Y, García A, Hernández A, Pérez J. 2011. Estudio de la variación del índice de color durante la conservación de la piña variedad Cayena Lisa a temperatura ambiente. Revista Ciencias Técnicas Agropecuarias 20: 12-16.

Giovannoni J. 2001. Molecular biology of fruit maturation and ripening. Annual Review of Plant Physiology and Plant Molecular Biology 52: 725-749. https://doi. org/10.1146/annurev.arplant.52.1.725

Jiménez B, Orea JM, Montero C, González A, Navas E, Slowing K, Gómez-Serranillos MP, Carretero E, De Martinis D. 2005. Resveratrol treatment controls microbial flora, prolongs shelf life, and preserves nutritional quality of fruit. Journal Agriculture Food Chemistry 53: 1523-1530. https://doi.org/10.1021/jft048426a

Jiménez-Zurita JO, Balois-Morales R, Alia-Tejacal I, Juárez-López P, Sumaya-Martínez MT, Bello-Lara JE.
2016. Caracterización de frutos de guanabana (Annona muricata L.) en Tepic, Nayarit, México. Revista Mexicana de Ciencias Agrícolas 7: 1261-1270.

Julián A. 2009. Propiedades físicas y químicas de tres variedades del fruto de Annona diversifolia. Trabajo especial de grado. Universidad Tecnológica de la Mixteca. Oaxaca, México. [Consultado 12 de octubre de 2019]. Disponible en: http://jupiter.utm.mx/ tesis_dig/10968. pdf.

Kusumaningrum D, Lee SH, Lee W-H, Mo C, Cho B-W. 2015. A review of technologies to prolong the shelf life of fresh tropical fruits in Southeast Asia. Journal of Biosystems Engineering 40: 345-358. https://doi. org/10.5307/JBE.2015.40.4.345

Lima MAC, Alves RE. 2011. Soursop (Annona muricata L.). In: Yahia E, editor. Postharvest biology and technology of tropical and subtropical fruits. Vol. 4. Mangosteen to white sapote. Elsevier, Amsterdam. P. 363-391.

Lima MAC, Alves RE, Filgueiras HAC, Enéas-Filho J. 2003. Comportamento respiratório e qualidade pós-colheita de graviola (Annona muricata L.) 'morada' sob temperatura ambiente. Revista Brasileira de Fruticultura 25: 49-52.

Lima MAC, Alves RE, Filgueiras HAC. 2006. Mudanças relacionadas ao amaciamento da graviola durante a maturação pós-colheita. Pesquisa Agropecuária Brasileira 41: 1707-1713. https://doi.org/10.101590/S01002004X2006001200004

Lima MAC, Alves RE, Filgueiras HAC. 2010. Comport amento respiratório e amaciamento de graviola (Annona muricata L.) após tratamentos pós-colheita com cera e 1-metilciclopropeno. Ciência e Agrotecnologia 34: 155-162. https://doi.org/10.1590/S141370542010000100020

Márquez CJ. 2009. Caracterización fisiológica, físico-química, reológica, nutracéutica, estructural y sensorial de la guanábana (Annona muricata L. cv. Elita). Tesis de Doctorado C. Universidad Nacional de Colombia. Medellín, Colombia. [Consultado 30 de marzo de 2019]. Disponible en: http://bdigital.unal.edu. co/1824/1/8740420.2009.pdf.

Márquez CJ, Villacorta V, Betancur DPY, Ciro HJ, Cartagena JR. 2012. Physiological and physico-chemical characterization of the soursop (Annona muricata L. cv. Elita). Revista de la Facultad Nacional de Agronomía-Medellín 65: 6477-6486.

Martínez-González M, Balois Morales R, Alia-Tejacal I, Cortes-Cruz M, Palomino-Hermosillo Y, López-Guzmán G. 2017. Postcosecha de frutos: maduración, ablandamiento y control transcripcional. Revista Mexicana de Ciencias Agrícolas Pub. Esp. Núm. 19: 4089-4101. https://doi.org/10.29312/remexca.v0i19.675

Montalvo GE, León FAE, Rea PH, Mata MDM, Tovar GB. 2014. Uso combinado de 1-meticiclopropeno y emulsiones de cera en la conservación de guanábana (Annona muricata L). Revista Brasileira Fruticultura 36, edição especial: 296-304. https://doi.org/10.1590/ S0100-29452014000500035 
Morales AA, Franco-Mora O, Castañeda-Vildózola Á, Morales-Rosales EJ. 2014. El efecto antisenescente del resveratrol reduce la tasa de ablandamiento poscosecha de chirimoya. Scientia Agropecuaria 5: 35-44.

Morales AA, Franco-Mora O, Castañeda-Vildózola Á, Morales-Rosales EJ. 2016. Efecto del resveratrol en frutos de chirimoya bajo simulación de transporte. Revista Mexicana de Ciencias Agrícolas 7: 127-132.

Moreno-Hernández CL, Sáyago-Ayerdi SG, García-Galindo HS, Mata-Montes de Oca M, Montalvo-González E. 2014. Effect of the application of 1-methylcyclopropene and wax emulsions on proximate analysis and some antioxidants of soursop (Annona muricata L.). The Scientific World Journal Article ID 896853: 1-7. http://dx.doi.org/10.1155/2014/896853

Pareek S, Yahia EM, Pareek OP, Kaushik RA. 2011. Postharvest physiology and technology of Annona fruits. Food Research International 44: 1741-1751. https://doi. org/10.1016/j.foodres.2011.02.016

Paull RE, Deputy JC, Chen NJ. 1983. Changes in organic acids, sugars and headspace vola- tiles during fruit ripening of soursop (Annona muricata L.). Journal of American Society Horticulture Science 108: 931-934.

Ploetz RC. 2003. Diseases of atemoya, cherimoya, soursop, sugar apple and related fruit crops. In: Ploetz RC, editor. Diseases of tropical fruit crops. CAB International, Wallingford. P. 21-34.

Reginato MG, Lizana A. 1980. Alteraciones detectadas en chirimoyas durante el almacenamiento. Investigación Agrícola 6: 97-101.

[SADER]. Secretaría de Agricultura y Desarrollo Rural [internet]. 2020. Estadística de la producción agrícola de 2018. Servicio de Información Agroalimentaria y Pesquera. [cited 2020 Ene 2]. Disponible en: http://infosiap.siap.gob.mx/gobmx/datosAbiertos_a.php.

Salomon-Castaño J, Franco-Mora O, Morales AA, Castañeda-Vildózola A, Villarreal-Fuentes JM, Sánchez-Pale JR. 2020. Improve of the preharvest application of resveratrol and 6-benzylaminopurine technique to reduce postharvest cherimoya fruit softness. Acta Horticulturae (Aceptado).

São José AR, Pires MM, De Freitas ALGE, Ribeiro DP, Pérez LAA. 2014. Atualidades e perpectivas das anonáceas no mondo. Revista Brasileira de Fruticultura 36: 86-93. https://doi.org/10.1590/S0100-29452014000500010

Siang LM, Ding P, Mohamend MTM. 2019. Response of 1-methylcyclopropene on postharvest quality of local soursop (Annona muricata L.). Sains Malasyana 48: 571579. http://dx.doi.org/10.17576/jsm-2019-4803-09

Systat Software, Inc. 2012. Systat, SigmaScan Pro, TableCurve 3D, PeakFit and AutoSignal. San José, USA.

Tovar-Gómez B, Mata-Montes de Oca M, García-Galindo HS, Montalvo-González E. 2011. Efecto de emulsiones de cera y 1-metilciclopropeno en la conservación poscosecha de guanabana. Revista Chapingo Serie Horticultura 17(Esp.1): 53-61.

Van Buren JP. 1986. Softening of cooked snap beans and other vegetables in relation to pectins and salts. In: Fi- shman ML, Jen JJ, editors. Chemistry and function of pectins. ACS Pub, Washington. P. 190-199.

Vignoni LA, Césari RM, Forte M, Mirábile ML. 2006. Determinación de índice de color en ajo picado. Información Tecnológica 17: 63-67. http://dx.doi.org/10.4067/ S0718-07642006000600011

Waterman PG, Mole S. 1994. Analysis of phenolic plant metabolites. Blackwell. Oxford, UK.

Worrell DB, Sean^Carrington CM, Huber DJ. 1994. Growth, maturation and ripening of soursop (Annona muricata L.) fruit. Scientia Horticulturae 57: 7-15. https:// doi.org/10.1016/0304-4238(94)90030-2

Yang X, Zhang Z, Joyce DC, Huang X, Xu L, Pang X. 2009. Characterization of chlorophyll degradation in banana and plantain during ripening at high temperature. Food Chemistry 114: 383-390. https://doi.org/10.1016/j. foodchem.2008.06.006

Zaicovski CB, Zimmerman T, Nora L, Nora FR, Silva JA, Rombaldi CV. 2008. Water stress increases cytokinin biosynthesis and delays postharvest yellowing of broccoli florets. Postharvest Biology and Technology 49: 436-439. https://doi.org/10.1016/j.postharvbio.2008.02.001

Zavaleta-Mancera HA, López-Delgado H, Loza-Tavera H, Mora-Herrera M, Trevilla-García C, Vargas-Suárez M, Ougham HJ. 2007. Cytokinin promotes catalase and ascorbate peroxidase activities and preserves the chloroplast integrity during dark-senescence. Journal of Plant Physiology 164: 1572-1582. https://doi.org/10.1016/j.jplph.2007.02.003

Zhu, LH, Van de Peppel A, Li XY, Welander M. 2004. Changes of leaf water potential and endogenous cytokinins in young apple trees treated with or without paclobutrazol under drought conditions. Scientia Horticulture 99: 133-141. https://doi.org/10.1016/S03044238(03)00089-X 